\title{
Low-Density Lipoprotein Cholesterol and Mortality in Older People
}

\author{
Valérie Tikhonoff, MD, ${ }^{*}$ Edoardo Casiglia, $M D,{ }^{*}$ Alberto Mazza, MD, PhD, ${ }^{*}$ Roberta Scarpa, MD, ${ }^{*}$ \\ Lutgarde Thijs, MSc, ${ }^{\dagger}$ Achille C. Pessina, MD, PhD, ${ }^{*}$ and Jan A. Staessen, MD, PhD. ${ }^{\dagger}$
}

OBJECTIVES: To investigate the role of low-density lipoprotein cholesterol (LDL-C) as a predictor of mortality in elderly subjects.

DESIGN: Population-based prospective cohort study.

SETTING: Two communities in northern Italy.

PARTICIPANTS: Three thousand one hundred twenty Caucasian subjects aged 65 and older recruited in for the Cardiovascular Study in the Elderly and followed up for 12 years.

MEASUREMENTS: Anthropometric measures: fasting plasma total cholesterol, triglyceride, high-density lipoprotein cholesterol, LDL-C, glucose, creatinine, and body mass index. Clinical measures: medical assessment, diabetes mellitus, hypertension, stroke, coronary disease, heart failure, and smoking and drinking habits. Vital status measures: death certificates from the Registry Office and causes of death according to the International Classification of Diseases. After plotting mortality rates using quartiles of LDL-C, relative hazard rates (RHRs) were calculated using multivariate Cox regression analyses. When the trend was nonlinear, the RHRs were further calculated for the 25th, 50 th, and 75 th percentiles of the distribution to confirm curvilinearity.

RESULTS: The distribution of risk of total mortality in women and of fatal heart failure in all subjects was curvilinear (non J-shaped), decreasing nonlinearly with LDL-C. For total mortality in men and cardiovascular mortality in both sexes, the relationship with LDL-C was J-shaped. The risk of fatal myocardial infarction was J-shaped in men, whereas it increased linearly with higher LDL-C in women. In both sexes, the association between stroke mortality and LDL-C was not significant.

From the * Department of Clinical and Experimental Medicine, University of Padova, Padova, Italy; and ${ }^{\dagger}$ Study Coordinating Center, Hypertension and Cardiovascular Rehabilitation Unit, Department of Molecular and Cardiovascular Research, University of Leuven, Leuven, Belgium.

Address correspondence to Dr. Edoardo Casiglia, Dipartimento di Medicina Clinica e Sperimentale, Università degli Studi di Padova, Via Giustiniani 2, I-35128 Padova, Italy. E-mail: edoardo.casiglia@unipd.it

DOI: 10.1111/j.1532-5415.2005.00492.x
CONCLUSION: This study adds to the uncertainty of the role of elevated levels of LDL-C as a risk factor for mortality in old people. J Am Geriatr Soc 53:2159-2164, 2005. Key words: elderly; mortality; ischemic heart disease; cardiovascular risk factors

$\mathrm{W}$ orldwide, populations are on a remarkable transition path from a state of high to low birth and death rates. One of every 10 persons is aged 60 and older; by 2050, the number of people within this age range will double. Serum lipids, in particular cholesterol and its subfractions, have long been recognized as important risk factors in middleaged subjects, ${ }^{1,2}$ but in an era of broadening indications to prescribe lipid-lowering drugs even at normal levels of serum cholesterol, ${ }^{3}$ prospective cohort studies ${ }^{4-9}$ on the role of serum lipids as risk factors in older people remain contradictory. Indeed, in some ${ }^{6,9,10}$ but not all studies, ${ }^{4,5}$ cholesterol predicted coronary complications, especially in men, whereas total and cardiovascular mortality were not $^{4,5}$ or were inversely ${ }^{6-9}$ associated with serum cholesterol. Furthermore, the risk associated with serum cholesterol differs according to ethnicity and geographic area. For example, rates of myocardial infarction (MI) are higher in northern than southern Europe. ${ }^{11}$ Moreover, patients randomized in clinical trials of lipid-lowering drugs were generally selected on the basis of multiple risk factors, and even in these high-risk patients, lowering cholesterol did not improve outcomes in women aged 40 to $79(\text { mean } 63)^{12}$ or 70 to 82 (mean 75$).{ }^{13}$

In the Cardiovascular Study in the Elderly (CASTEL), elderly people were recruited from a general Italian population, and mortality was monitored from 1983 to 1995. The roles of low-density lipoprotein cholesterol (LDL-C) and other serum lipids were investigated as predictors of allcause and cardiovascular mortality while allowing for other risk factors and nonlinear relationships.

\section{METHODS}

From 1983 to 1985 , 3,120 subjects aged 65 and older, representing $73 \%$ of the elderly inhabitants of the 
northern Italian towns of Castelfranco and Chioggia, were enrolled. The study was conducted according to the principles outlined in the Helsinki Declaration for investigation in human subjects. The CASTEL ethics review committee approved the protocol. ${ }^{14}$ Participants gave informed consent.

At baseline, trained doctors who were part of the ad hoc team measured sphygmomanometric blood pressure (phase 5 diastolic) in triplicate at 5-minute intervals and determined pulse rate. These procedures were repeated twice at 1-month intervals. For the present analysis, the blood pressure readings of the last visit were averaged. Body mass index (BMI) was calculated as body weight $(\mathrm{kg})$ divided by height squared $(\mathrm{m})$. A standardized questionnaire was administered to collect information on each subject's medical history, use of medications, and smoking and drinking habits. After an overnight fast, venous blood was collected for measurement of blood glucose, serum uric acid, serum creatinine, and serum lipids, including total and high-density lipoprotein cholesterol (HDL-C) and triglycerides. LDL-C was calculated using Friedewald's algorithm. ${ }^{15}$ Diabetes mellitus was defined as a fasting blood glucose of $7.0 \mathrm{mmol} / \mathrm{L}(126 \mathrm{mg} / \mathrm{dL})$ or higher on two separate occasions or as receiving treatment with antidiabetic agents. Subjects were followed for 12 years. ${ }^{16}$ Death certificates were obtained from the Registry Office. If the cause of death could not be established with certainty from the death certificate, the diagnosis was ascertained via consultation with the family doctors' files, hospital, and retirement home clinical files and by asking first of kin. A trained research physician coded causes of death according to the International Classification of Diseases, Ninth Revision. Codes 398.91; 402.0; 410-414; 414.1, 8, and 9; 415.1; 428.0-3 and 9; 429.1, 2, and 7; 430-438; 444; and 492.2 were used to define cardiovascular mortality. Mortality was defined as cardiovascular when due to coronary heart disease, stroke, heart failure, arrhythmias, or pulmonary embolism and as noncardiovascular when due to other causes. No cause of death was missed.

To avoid bias in the process of record review, those who analyzed the causes of death were blind to subjects' LDL-C level. Stroke was a neurological deficit leading to death with no apparent cause other than vascular. Cardiovascular mortality included sudden death, stroke, MI, heart failure, and other cardiovascular disorders. History of cardiovascular events (MI, angina pectoris, heart failure, intermittent claudication, and stroke) was also recorded. Subjects with at least one of the following items at baseline-Minnesota code $1.1,1.2$, or 1.3 (if absent 6.4 .1 ); 4.1 or 4.4 (if absent 6.4.1, 7.1.1, and 7.2.1); or 5.1, 5.2, 5.3, or 5.4 (if absent 6.4.1, 7.1.1, 7.2.1, and 7.4), positive myocardial scintigraphy, positive stress test, history of MI confirmed by hospital files, history of angina pectoris confirmed by hospital or physician's files, or appropriate antianginal chronic treatment - were labeled as having historical coronary heart disease. Subjects having clear and permanent neurological consequences or a positive history confirmed by hospital or physician's files or a positive computed tomography scan were considered to have had a stroke. Subjects with enlarged heart according to chest $\mathrm{x}$-ray, dyspnea, or peripheral edema were considered to have congestive heart failure.
None of the 3,120 subjects recruited for the study had dementia (a condition that could reduce life expectancy and confound the relationship between LDL-C and death), because people with dementia would have been unable to answer the Rose's questionnaire.

For statistical analysis, SAS software, version 8.1 (SAS Institute, Inc., Cary, NC) was used. Means and proportions were compared using analysis of variance and a multiple means test and proportions using the chi-square test. Death rates adjusted for age were first plotted using the direct method by quartiles of serum lipid distributions in women and men separately. Risk factors affecting the incidence of mortality were then identified using a stepwise Cox regression procedure, terminating when all regression coefficients in the model were statistically significant at the $5 \%$ level. Baseline characteristics considered for entry into the Cox model were age, systolic and diastolic blood pressure, pulse rate, BMI, historical cardiovascular events, current smoking and alcohol intake, a history of diabetes mellitus, and serum concentrations of creatinine and uric acid. Using Cox regression, whether the addition of a squared term of serum lipid concentration significantly added to the risk prediction was tested. In case of curvilinear fit of the risk function, relative hazard rates (RHRs) are not constant over the range of serum lipid concentration. For such relationships, RHRs with $95 \%$ confidence intervals (CIs) for a 1-mmol LDL-C increase above the $25 \mathrm{th}$, 50th, and 75 th percentiles of the distribution were reported.

\section{RESULTS}

The study participants included 1,887 women and 1,233 men. Mean age \pm standard deviation was $73.8 \pm 5.3$, and BMI averaged $26.6 \pm 4.0 \mathrm{~kg} / \mathrm{m}^{2}$. Prevalence of heart disease was $54.0 \%(66.4 \%$ in men and $45.9 \%$ in women, $P<.001)$. The 5 th to 95 th percentile intervals of serum total cholesterol and LDL-C ranged from 3.9 to $7.6 \mathrm{mmol} / \mathrm{L}$ (151$293 \mathrm{mg} / \mathrm{dL})$ and from 2.0 to $5.2 \mathrm{mmol} / \mathrm{L}(77-201 \mathrm{mg} / \mathrm{dL})$, respectively. Women had higher systolic (161.8 vs $156.8 \mathrm{mmHg})$ and diastolic $(89.4 \mathrm{vs} 87.7 \mathrm{mmHg})$ blood pressure; faster pulse rate ( 77.6 vs 73.6 beats per minute); and higher serum concentrations of total cholesterol (5.9 vs $5.4 \mathrm{mmol} / \mathrm{L} ; 228$ vs $208 \mathrm{mg} / \mathrm{dL}$ ), LDL-C (3.6 vs $3.4 \mathrm{mmol} / \mathrm{L}$; 139 vs $131 \mathrm{mg} / \mathrm{dL}$ ), HDL-C (1.6 vs $1.4 \mathrm{mmol} / \mathrm{L} ; 62$ vs $54 \mathrm{mg} / \mathrm{dL})$, and triglycerides $(1.5 \mathrm{vs} 1.4 \mathrm{mmol} / \mathrm{L} ; 133$ vs $124 \mathrm{mg} / \mathrm{dL})$ than men $(P<.001)$. Serum creatinine $(78.7$ vs $90.2 \mathrm{mmol} / \mathrm{L} ; 0.89$ vs $1.02 \mathrm{mg} / \mathrm{dL}$ ) and uric acid (303 vs $334 \mathrm{mmol} / \mathrm{L} ; 5.1$ vs $5.6 \mathrm{mg} / \mathrm{dL})$ were lower $(P<.001)$ in women than men. Because of these sex differences, women and men were analyzed separately.

In men, systolic blood pressure was $3.1 \%, 3.1 \%$, and $3.4 \%(P<.05)$ higher in the second, third, and fourth quartiles of LDL-C than in the first $(153.1 \pm 23.0 \mathrm{mmHg})$. In women, systolic blood pressure was $2.8 \%, 2.4 \%$, and $2.8 \%$ $(P<.05)$ higher in the second, third, and fourth quartiles, respectively, than in the first $(158.6 \pm 24.5 \mathrm{mmHg})$, and diastolic blood pressure was $2.4 \%, 2.4 \%$, and $2.5 \%$ $(P<.05)$ higher in the second, third, and fourth quartiles, respectively, than in the first $(87.9 \pm 11.8 \mathrm{mmHg})$. In both sexes, BMI was significantly higher in the fourth than in the first quartile of LDL-C $(5.9 \%, P<.05$, in men; $3.0 \%$, $P<.05$, in women), whereas HDL-C was lower $(-13.3 \%$, 


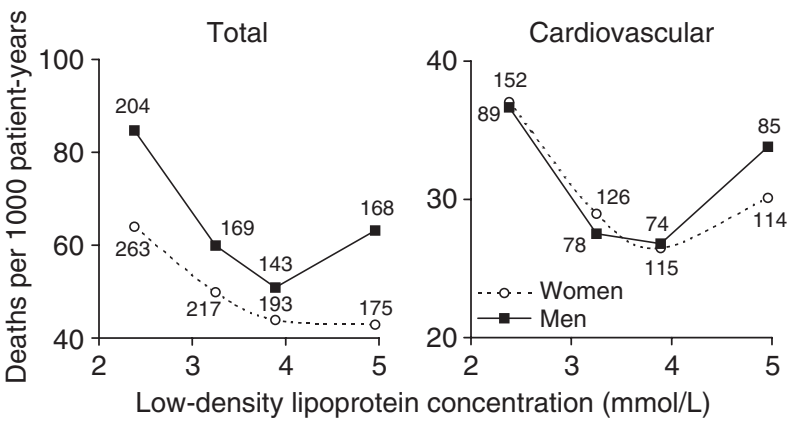

Figure 1. Sex-specific and age-adjusted rates of total and cardiovascular mortality by quartiles of serum low-density lipoprotein cholesterol at baseline. The number of deaths is given for each quartile. Conversion factor to conventional units is 38.6.

$P<.05$, in men; $-6.2 \%, P<.05$, in women). No differences across quartiles of LDL-C were observed for the other characteristics in either sex.

Median follow-up was 11.1 years (range 0.02-12.0). Death rates were first evaluated according to quartiles of serum LDL-C. In both sexes, the age-adjusted death rates in relation to serum LDL-C showed a curvilinear or U-shaped pattern for total and cardiovascular mortality (Figure 1) and an inverse association for deaths from heart failure (Figure 2).

Stepwise Cox regression identified age, systolic blood pressure, history of diabetes mellitus and cardiovascular disease, and serum uric acid concentration as consistent predictors of one or more fatal outcomes in women and men. Furthermore, in men and women, coronary and total mortality increased with higher serum creatinine concentration. In addition, all-cause mortality was inversely and independently correlated with BMI. In men but not women, higher pulse rate and current smoking were significant and independent predictors of mortality. All RHRs were therefore adjusted for age, systolic blood pressure, history of diabetes mellitus and cardiovascular disease, and serum uric acid concentration and in men also for pulse rate and current smoking. RHRs for MI and total mortality were additionally adjusted for serum creatinine concentration and for BMI and serum creatinine concentration, respectively.
Cox regression confirmed that, in women and men (Tables 1 and 2), deaths from all and cardiovascular causes and those from heart failure were independently and curvilinearly related to serum LDL-C. The nadirs of these relations were from 0.62 to $1.02 \mathrm{mmol} / \mathrm{L}(24-39 \mathrm{mg} / \mathrm{dL})$ higher in women than men. In women (Figure 1 and Table 1 ), total mortality decreased with higher serum LDL-C, but the decline in risk was more pronounced at low (25th percentile) than high (75th percentile) serum LDL-C levels. The Cox model revealed a similar inverse association with LDL-C for mortality from heart failure in women (Figure 2 and Table 1) and men (Figure 2 and Table 2). For total mortality in men (Figure 1 and Table 2) and for cardiovascular mortality in women and men (Figure 1 and Tables 1 and 2), the curvilinear fit between these fatal outcomes and LDL-C indicated that, with increasing LDL-C levels, the risk declined at the lower end of the LDL-C distribution, whereas at the upper end, the opposite trend was observed. In men, the risk of dying from MI increased curvilinearly with higher serum LDL-C. The probability of an event increased more at higher than lower LDL-C levels (Figure 2 and Table 2). In women, the risk of MI also increased with higher serum LDL-C, but this relationship was linear. In women and men alike, the association between stroke mortality and LDL-C was not significant (Figure 2 and Tables 1 and 2).

For total serum cholesterol, the model fit in Cox regression and the RHRs were similar to those observed for LDL-C (data not shown). Mortality tended to be linearly and inversely correlated with serum HDL-C, but the relationship was only significant for death from $\mathrm{MI}$ in women $(\mathrm{RHR}=0.59,95 \% \mathrm{CI}=0.35-0.99, P=.046)$.

Serum triglyceride concentration was a linear and independent predictor of mortality. In men, the adjusted RHR for a $0.5-\mathrm{mmol} / \mathrm{L}$ increase in triglycerides was $1.20(95 \%$ $\mathrm{CI}=1.10-1.32, P<.001)$ for total mortality, $1.17(95 \%$ $\mathrm{CI}=1.03-1.33, P=.02)$ for cardiovascular mortality, 1.34 $(95 \% \mathrm{CI}=1.03-1.76, P=.03)$ for mortality from MI, and $1.26(95 \% \mathrm{CI}=1.05-1.52, P=.02)$ for heart failure mortality. In women, the relationship was significant for MI mortality only $(1.18,95 \% \mathrm{CI}=1.00-1.40, P=.047)$.

The findings for LDL-C were not materially altered when the Cox models also accounted for other lipid fractions such as HDL-C or serum triglycerides.

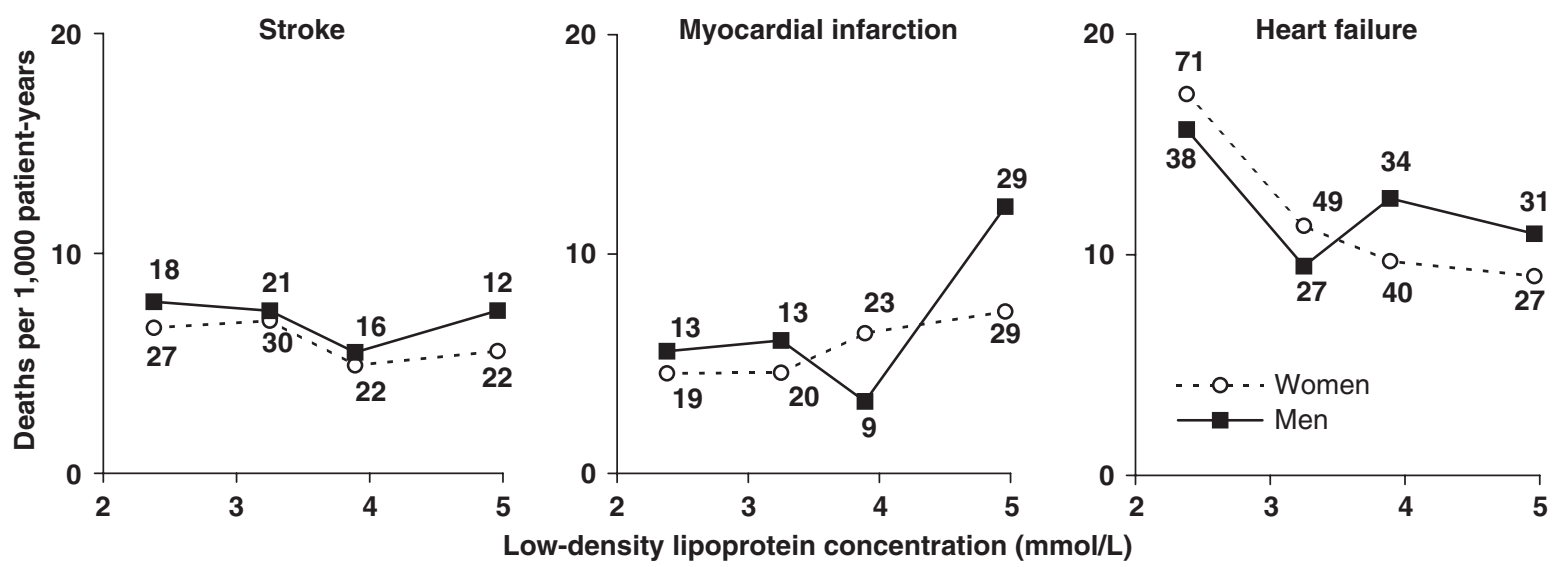

Figure 2. Sex-specific and age-adjusted rates of categorized cardiovascular mortality by quartiles of serum low-density lipoprotein cholesterol at baseline. The number of deaths is given for each quartile. Conversion factor to conventional units is 38.6. 
Table 1. Association Between Mortality and Serum Low-Density Lipoprotein Cholesterol (LDL-C)* Concentration in Women

\begin{tabular}{|c|c|c|c|c|c|c|c|}
\hline \multirow[b]{2}{*}{ Endpoint } & \multirow[b]{2}{*}{$\mathrm{N}$} & \multicolumn{2}{|c|}{ Model } & \multirow[b]{2}{*}{ Nadir (mmol/L) } & \multicolumn{3}{|c|}{$\begin{array}{c}\text { Adjusted }^{\dagger} \text { Relative Hazard Ratio (95\% Confidence Interval) } \\
\text { for } 1-\mathrm{mmol} \text { Increase in LDL-C }\end{array}$} \\
\hline & & Fit & P-value & & $\mathrm{P}_{25}=2.9 \mathrm{mmol} / \mathrm{L}$ & $\mathrm{P}_{50}=3.6 \mathrm{mmol} / \mathrm{L}$ & $\mathrm{P}_{75}=4.2 \mathrm{mmol} / \mathrm{L}$ \\
\hline Total $^{\ddagger}$ & 848 & $\mathrm{CL}$ & .03 & 4.86 & $0.84(0.79-0.91)$ & $0.92(0.85-0.98)$ & $0.98(0.89-1.08)$ \\
\hline Cardiovascular & 507 & $\mathrm{CL}$ & .03 & 4.26 & $0.88(0.81-0.97)$ & $0.97(0.89-1.06)$ & $1.06(0.94-1.18)$ \\
\hline Myocardial infarction ${ }^{\S}$ & 91 & $\mathrm{~L}$ & .04 & - & - & $1.23(1.01-1.50)$ & - \\
\hline Stroke & 101 & $\mathrm{~L}$ & .31 & - & - & $0.90(0.73-1.11)$ & - \\
\hline Heart failure & 197 & $\mathrm{CL}$ & .04 & 4.70 & $0.78(0.68-0.90)$ & $0.89(0.77-1.02)$ & $0.99(0.82-1.20)$ \\
\hline
\end{tabular}

* Conversion factor to conventional units for LDL-C is 38.6.

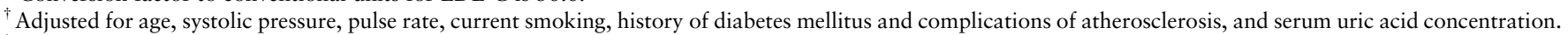

* Additionally adjusted for body mass index and serum creatinine.

$\$$ Additionally adjusted for serum creatinine.

$\mathrm{P}_{25}, \mathrm{P}_{50}, \mathrm{P}_{75}=25$ th, 50th, and 75 th percentiles of the serum LDL-C distribution; $\mathrm{CL}=$ curvilinear fit; $\mathrm{L}=$ linear fit.

\section{DISCUSSION}

The key finding of this study was that, in older subjects with low use of lipid-lowering drugs representative of the general Italian population, serum LDL-C behaved as a multifaceted and predominantly nonlinear predictor of cardiovascular and all-cause mortality. The risk of total mortality in women and fatal heart failure in both sexes decreased with higher LDL-C, the steepest decline occurring at the lower end of the LDL-C distribution. For total mortality in men and cardiovascular mortality in women and men, the relationship with LDL-C was U-shaped. Furthermore, LDL-C was a consistent predictor of MI. In men, the risk of coronary mortality increased more at high than low LDL-C levels, whereas in women, the risk increase was constant over the range of LDL-C.

Serum cholesterol is a strong predictor of coronary heart disease and all-cause mortality in middle-aged populations. ${ }^{1,2,10}$ The Multiple Risk Factor Intervention Trial showed a curvilinear increment in the death rate of coronary heart disease in 361,662 men aged 35 to 57 when total serum cholesterol exceeded $4.7 \mathrm{mmol} / \mathrm{L}(181 \mathrm{mg} / \mathrm{dL}) .{ }^{2}$ The evidence with regard to the risk associated with total cholesterol or LDL-C in older populations is conflicting. ${ }^{4,6,8}$ In the trial conducted by the European Working Party on High
Blood Pressure in the Elderly (aged $\geq 60,70 \%$ women), ${ }^{17}$ serum cholesterol measured at randomization was independently and inversely correlated with total mortality. The correlations between cardiovascular and cardiac mortality and serum total cholesterol were not significant. ${ }^{17}$ All factors being equal, an increase in serum total cholesterol was associated with a 1 -year prolongation of survival. ${ }^{17}$ In the Framingham Heart Study, ${ }^{18}$ the risk ratio of coronary heart disease between the highest and lowest quartiles of total serum cholesterol was 2.2 for men aged 50 and older, compared with 3.6 for younger men. One study calculated pooled risk estimates across 22 cohort studies. ${ }^{19}$ The relative risk ratios of fatal coronary heart disease in relationship to total cholesterol and LDL-C were statistically significant across a broad age range and into old age (65), although as in the Framingham Heart Study, ${ }^{18}$ the relative risk weakened with advancing age, especially in women. Several other prospective population studies reported positive and significant correlations between the incidence of coronary events and serum total cholesterol or LDL-C in older age groups. ${ }^{10,11,19,20}$ In contrast, many researchers failed to demonstrate a positive association between coronary risk and serum cholesterol level in older subjects. ${ }^{4,5}$ This was probably attributable to previous selec-

Table 2. Association Between Mortality and Serum Low-Density Lipoprotein Cholesterol (LDL-C)* Concentration in Men

\begin{tabular}{|c|c|c|c|c|c|c|c|}
\hline \multirow[b]{2}{*}{ Endpoint } & \multirow[b]{2}{*}{$N$} & \multicolumn{2}{|c|}{ Model } & \multirow[b]{2}{*}{ Nadir (mmol/L) } & \multicolumn{3}{|c|}{$\begin{array}{c}\text { Adjusted }^{\dagger} \text { Relative Hazard Ratio (95\% Confidence Interval) } \\
\text { for 1-mmol Increase in LDL-C }\end{array}$} \\
\hline & & Fit & $P$-value & & $\mathrm{P}_{25}=2.7 \mathrm{mmol} / \mathrm{L}$ & $\mathrm{P}_{50}=3.3 \mathrm{mmol} / \mathrm{L}$ & $\mathrm{P}_{75}=3.9 \mathrm{mmol} / \mathrm{L}$ \\
\hline Total ${ }^{\ddagger}$ & 684 & $\mathrm{CL}$ & .001 & 3.84 & $0.84(0.77-0.92)$ & $0.99(0.91-1.07)$ & $1.16(1.05-1.29)$ \\
\hline Cardiovascular & 327 & $\mathrm{CL}$ & .001 & 3.64 & $0.90(0.79-1.02)$ & $1.03(0.92-1.16)$ & $1.19(1.02-1.38)$ \\
\hline Myocardial infarction $\$$ & 64 & $\mathrm{CL}$ & .04 & 2.44 & $1.20(0.88-1.63)$ & $1.41(1.11-1.79)$ & $1.66(1.31-2.10)$ \\
\hline Stroke & 67 & L & .14 & - & - & $0.80(0.60-1.07)$ & - \\
\hline Heart failure & 130 & $\overline{\mathrm{CL}}$ & .03 & 3.72 & $0.84(0.70-1.03)$ & $1.10(0.86-1.21)$ & $1.22(0.98-1.54)$ \\
\hline
\end{tabular}

* Conversion factor to conventional units for LDL-C is 38.6.

${ }^{\dagger}$ Adjusted for age, systolic pressure, pulse rate, current smoking, history of diabetes mellitus and complications of atherosclerosis, serum uric acid concentration, pulse rate, and current smoking.

${ }^{\ddagger}$ Additionally adjusted for body mass index and serum creatinine.

$\S_{\text {Additionally adjusted for serum creatinine. }}$

$\mathrm{P}_{25}, \mathrm{P}_{50}, \mathrm{P}_{75}=25$ th, 50th, and 75th percentiles of the serum LDL-C distribution; $\mathrm{CL}=$ curvilinear fit; $\mathrm{L}=$ linear fit. 
tion, in the decades before the study, of subjects doomed to survive despite cholesterol thanks to nonlipidic favorable risk patterns. In elderly and very elderly populations, the incidence of stroke rises exponentially with age so that, in septuagenarians and octogenarians, stroke outweighs MI as the major complication associated with blood pressure. ${ }^{21}$ As in the present study, serum cholesterol is often reported as an inconsistent predictor of stroke, probably because it is inversely associated with the risk of hemorrhagic stroke. ${ }^{20,22}$

In the major primary ${ }^{23,24}$ and secondary ${ }^{25,26}$ prevention trials of 3-hydrony-3 methylglutaryl coenzyme A reductase inhibitors (statins) published before 2000, mean serum LDL-C concentration at randomization ranged from $3.6 \mathrm{mmol} / \mathrm{L}^{26}$ to $5.0 \mathrm{mmol} / \mathrm{L}^{23}(139-193 \mathrm{mg} / \mathrm{dL})$. Women represented only $7.5 \%$ of the participants enrolled in the primary prevention trials. ${ }^{27}$ The secondary prevention studies included just subjects with established coronary heart disease ${ }^{25}$ or a history of MI. ${ }^{26}$ Of the current study participants, 912 and 172 women $(48.3 \%$ and $9.1 \%)$ and 462 and 62 men $(37.8 \%$ and $5.1 \%)$ had serum LDL-C levels in excess of $3.6 \mathrm{mmol} / \mathrm{L}$ or $5.0 \mathrm{mmol} / \mathrm{L}$ ( 139 or $193 \mathrm{mg} /$ $\mathrm{dL})$, respectively. Of subjects with a serum LDL-C concentration higher than $3.6 \mathrm{mmol} / \mathrm{L}(139 \mathrm{mg} / \mathrm{dL}), 20$ women $(2.2 \%)$ and 39 men $(8.4 \%)$ had a history of ischemic heart disease at baseline.

In general, the early statin trials suffered from age and sex bias, having been mainly conducted in middle-aged male populations. ${ }^{27}$ Extrapolations from these trials to older people and women needed further evaluation. Recently published intervention studies have addressed these concerns; $3,12,13,28$ of 47,000 randomized participants, $15,075(32.1 \%)$ were female, and 11,610 (24.7\%) were aged 70 and older. Overall, these trials included more than $60 \%$ of subjects aged 60 and older. Unfortunately, their results can also not be extrapolated to the overall elderly population or to ethnic groups with low mortality from coronary heart disease. Indeed, these trials selectively enrolled high-risk patients with preexisting vascular disease (coronary, cerebral, or peripheral), with multiple risk factors such as old age, smoking, hypertension, or diabetes mellitus, or with both sets of prognosis-aggravating characteristics. Use of statins could therefore be useful in those selected elderly subjects. Thus, even these recent trials cannot be viewed as being representative of a general older population as described in the present report, in which cholesterol behaved as a multifaceted risk factor.

This study must be interpreted within the context of its limitations. First, the study was conducted in an Italian population, so the results can probably be extrapolated to Mediterranean people only. Serum LDL-C levels were not measured but were calculated according to Friedewald's formula. ${ }^{15}$ At baseline, only a single measurement of the serum lipids was obtained, so regression dilution bias could not be corrected for. ${ }^{29}$ This study included only fatal outcomes. The available medical records did not allow distinguishing between hemorrhagic and ischemic stroke. Assessment of dementia was incomplete, but recent data have shown that this is not an important confounding factor, because an increased risk of death associated with low LDL-C can be also found after excluding participants with dementia. ${ }^{8}$ Nevertheless, the study participants represented more than $70 \%$ of the elderly inhabitants of two Italian towns. Moreover, recent trials of lipid-lowering drugs ${ }^{3,29}$ also used total mortality as their primary outcome, because it incorporates cancer, can be easily validated, and is the most meaningful endpoint possible in any study. In addition, the Heart Protection Study demonstrated similar effects of lipid lowering on fatal and nonfatal cardiovascular outcomes. ${ }^{3}$

In conclusion, in unselected older subjects of Italian extraction, serum LDL-C concentration is a multifaceted risk factor. Results from trials of lipid-lowering drugs in high-risk patients cannot be extrapolated to unselected elderly populations without further evidence.

\section{ACKNOWLEDGMENTS}

Financial Disclosure: V. Tikhonoff, E. Casiglia, A. Mazza, R. Scarpa, L. Thijs, A.C. Pessina, and J.A. Staessen have no financial disclosures to make in relation to this paper.

Author Contributions: V. Tikhonoff performed the analysis, took part in the interpretation of the results, and prepared the final version of the manuscript. E. Casiglia designed and coordinated the CASTEL study, took part in the interpretation of the results, and prepared the final version of the manuscript. A. Mazza and R. Scarpa participated in the acquisition of data and the construction of the database. L. Thijs gave statistical advice, took part in the interpretation of the results, and prepared the final version of the manuscript. A.C. Pessina took part in the interpretation of the results and reviewed the manuscript. J. A. Staessen took part in the interpretation of the results and prepared the final version of the manuscript.

Sponsor's Role: No sponsor.

\section{REFERENCES}

1. Anderson KM, Castelli WP, Levy D. Cholesterol and mortality: 30 years of follow-up from the Framingham study. JAMA 1987;257:2176-2180.

2. Martin MJ, Hulley SB, Browner WS et al. Serum cholesterol, blood pressure, and mortality: Implications from a cohort of 361,662 men. Lancet 1986;2:933-936.

3. Heart Protection Study Collaborative Group. MRC/BHR Heart Protection Study of cholesterol lowering with simvastatin in 20,536 high-risk individuals: A randomised placebo-controlled trial. Lancet 2002;360:7-22.

4. Krumholz HM, Seeman TE, Merrill SS et al. Lack of association between cholesterol and coronary heart disease mortality and morbidity and all-cause mortality in persons older than 70 years. JAMA 1994;272:1335-1340.

5. Zimetbaum P, Frishman WH, Ooi WL et al. Plasma lipids and lipoproteins and the incidence of cardiovascular disease in the very elderly: The Bronx Aging Study. Arterioscler Thromb 1992;12:416-423.

6. Schatz IJ, Masaki K, Yano K et al. Cholesterol and all-cause mortality in elderly people from the Honolulu Heart Program: A cohort study. Lancet 2001;358:351-355.

7. Brescianini S, Maggi S, Farchi G et al. for the ILSA Group. Low total cholesterol and increased risk of dying: Are low levels clinical warning signs in the elderly? Results from the Italian Longitudinal Study on Aging. J Am Geriatr Soc 2003;51:991-996.

8. Schupf N, Costa R, Luchsinger J et al. Relationship between plasma lipids and all-cause mortality in nondemented elderly. J Am Geriatr Soc 2005;53:219_ 226.

9. Psaty BM, Anderson M, Kronmal RA et al. The association between lipid levels and the risks of incident myocardial infarction, stroke, and total mortality: The Cardiovascular Health Study. J Am Geriatr Soc 2004;52:1639_ 1647.

10. Benfante R, Reed W. Is elevated serum cholesterol level a risk factor for coronary heart disease in the elderly? JAMA 1990;263:393-396.

11. Houterman S, Verschuren MWM, Giampaoli S et al. Total but not high-density lipoprotein cholesterol is consistently associated with coronary heart dis- 
ease mortality in elderly men in Finland, Italy, and the Netherlands. Epidemiology 2000;11:327-332.

12. Sever PS, Dahlöf B, Poulter NR et al. Prevention of coronary and stroke events with atorvastatin in hypertensive patients who have average or lower-thanaverage cholesterol concentrations, in the Anglo-Scandinavian Cardiac Outcomes Trial- Lipid Lowering Arm (ASCOT-LLA): A multicentre randomized controlled trial. Lancet 2003;361:1149-1158.

13. Shepherd J, Blauw GJ, Murphy MB et al. Pravastatin in elderly individuals at risk of vascular disease (PROSPER): A randomized controlled trial. Lancet 2002;360:1623-1630.

14. Casiglia E, Spolaore P, Mormino P et al. The CASTEL project (CArdiovascular STudy in the ELderly). Protocol, study design, and preliminary results of the initial survey. Cardiologia 1991;36:569-576.

15. Friedewald WT, Levy RI, Frederickson DS. Estimation of the concentration of low-density lipoprotein cholesterol in plasma, without use of the preparation ultracentrifuge. Clin Chem 1972;18:499-502.

16. Casiglia E, Palatini P. Cardiovascular risk factors in the elderly. J Hum Hypertens 1998;12:575-581.

17. Staessen J, Amery A, Birkenhager W et al. Is a high serum cholesterol level associated with longer survival in elderly hypertensives? J Hypertens 1990;8:755-761.

18. Kannel WB, Castelli WP, McNamara PM. Serum cholesterol, lipoproteins, and the risk of coronary heart disease. The Framingham Study. Ann Intern Med 1971;74:1-12.

19. Manolio TA, Pearson TA, Wenger NK et al. Cholesterol and heart disease in older persons and women. Review of an NHLBI Workshop. Ann Epidemiol 1992;2:161-176.

20. Simons LA, Simons J, Friedlander Y et al. Cholesterol and other lipids predict coronary heart disease and ischaemic stroke in the elderly, but only in those below 70 years. Atherosclerosis 2001;159:201-208.
21. Bonita R. Epidemiology of stroke. Lancet 1992;339:342-344.

22. Okamura T, Kadowaki T, Hayakawa T et al. What cause of mortality can we predict by cholesterol screening in the Japanese general population? J Intern Med 2003;253:169-180.

23. Shepherd J, Cobbe SM, Ford I et al. for the West of Scotland Coronary Prevention Study Group. Prevention of coronary heart disease with pravastatin in men with hypercholesterolemia. N Engl J Med 1995;333:1301-1307.

24. Downs JR, Clearfield $M$, Weis $S$ et al. for the AFCAPS/TexCAPS research group. Primary prevention of acute coronary events with lovastatin in men and women with average cholesterol level. JAMA 1998;279:16151622.

25. Scandinavian Simvastatin Survival Study Group. Randomised trial of cholesterol lowering in 4444 patients with coronary heart disease (4S). Lancet 1994;344:1383-1389.

26. Sacks FM, Pfeffer MA, Moyee LA et al. For the cholesterol and recurrent events trial investigators. The effect of pravastatin on coronary events after myocardial infarction in patients with average cholesterol levels. N Engl J Med 1996;335:1001-1009.

27. Bandyopadhyay S, Bayer AJ, O'Mahony MS. Age and gender bias in statin trials. Q J Med 2001;94:127-132.

28. The ALLHAT Officers and Coordinators for the ALLHAT Collaboration Research Group. The Antihypertensive and Lipid-Lowering Treatment to Prevent Heart Attack Trial. Major outcomes in moderately hypercholesterolemic, hypertensive patients randomized to pravastatin vs usual care: The antihypertensive and lipid-lowering treatment to prevent heart attack trial (ALLHATLLT). JAMA 2002;288:2998-3007.

29. Emberson JR, Whincup PH, Morris RW et al. Re-assessing the contribution of serum total cholesterol, blood pressure and cigarette smoking to the aetiology of coronary heart disease: Impact of regression dilution bias. Eur Heart J 2003;24:1719-1726. 